\title{
Integrating Origin-Destination Survey and Stochastic User Equilibrium: A Case Study for Route Relocation
}

\author{
Deo Chimba ${ }^{*}$, Daniel Emaasit, Boniphace Kutela \\ Department of Civil Engineering, Tennessee State University, Nashville, USA \\ Email: dchimba@tnstate.edu
}

Received June 22, 2012; revised July 20, 2012; accepted August 2, 2012

\begin{abstract}
The paper analyses integrating Origin-Destination (O-D) survey results with Stochastic User Equilibrium (SUE) in traffic assignment. The two methods are widely used in transportation planning but their applications have not yet fully integrated. While O-D gives a generalized trip patterns, purpose and characteristics, SUE provides optimal trip distributions using the characteristics found in O-D survey. The paper utilized O-D and SUE in route relocation study for the town of Coamo in Puerto Rico. The O-D survey was used initially in studying possible trip distribution and assignment for the new route. Initial distribution and assignment of traffic to the existing roadway networks and the proposed route were allocated utilizing the O-D survey findings. The SUE was then used to optimize the assignments considering roadway characteristics such as number of lanes, capacity limits, free flow speed, signal spacing density, travel time and gasoline cost. The travel time was optimized through the Bureau of Public Roads (BPR) equation found in 2000 HCM. The optimal trips found from the SUE were then used to propose the final alignment of the new route. Traffic assignment from the SUE was slightly different from those initially assigned using O-D, indicating there was optimization. The assignment on new route was increased by $13.8 \%$ from the one assigned using O-D while assignment on the existing link was reduced by $22 \%$.
\end{abstract}

Keywords: Route Relocation; Origin-Destination; Stochastic User Equilibrium

\section{Introduction and Background}

Knowledge of the travel patterns for a defined jurisdiction or roadway network is an important aspect in transportation planning [1]. The patterns may include vehicle classifications, trip purposes, travel time, age differentiations, life styles and vehicle occupancy among others. The information can be used for different purposes including traffic impact studies, corridor and area planning, zoning, master plans, traffic projection and traffic assignments. There are different methodologies used in studying traffic patterns, one of them being Origin and Destination (O-D) survey. While some studies use O-D as a stand-alone approach in traffic pattern studies, some have combined the information from O-D report with other supporting traffic data to facilitate the findings and conclusions. For instance, O-D can be used in demand estimation using turning movement counts [2]. There are some studies whose objectives can be fulfilled with the O-D survey information only, but most of them will need supporting data or analysis in order to draw practical conclusions. Taking example of the O-D survey giving the percentage of trips from city A to city B, in rare cases

"Corresponding author. the same survey will give the route assignment used by the interviewed travelers. In this case, while the percentage of trips from city A to city B will be obtained for planning purposes, supporting information related to the route assignments will be needed. In other words, the results from O-D study will need supporting analysis to make final recommendations. The O-D study gives the details of what type of trips in terms of purpose at origin and destination are made by the travelers. Through O-D, one can determine which among home-based, education, shopping, recreational or any other trip purpose are dominant in the area. In case of route relocation study, diversions, road expansion and other similar kind of projects, O-D survey becomes not a stand-alone but a supporting document [3].

\subsection{Study Objectives}

This study therefore combines the O-D survey with the traffic assignment analysis using stochastic user equilibrium in route relocation study. The objective is to evaluate which approaches (between utilizing O-D survey only, SUE only or integrated O-D and SUE) yield the optimal and desired results. The desired results in this case are 
defined in terms of attributes such as traffic volumes which eventually lead to choosing number of lanes and intersection configurations. The project required the formulation and evaluation of a number of alternatives and combination of alternatives for the selection of a new improved roadway connection between the PR-52 interchange and the central area of the town of Coamo in Puerto Rico, Figure 1. PR-52 is the 4-lane limited access highway running E-W just south of the town connecting to the other major cities to the west, south and east. The existing roadway system PR-153 and PR-545 are the main roadways connecting central Coamo and PR-52. PR-545 connects PR-52 to PR-14, which runs to the downtown area. Currently PR-153 is operating beyond the capacity while PR-545 is substandard. The proposed new alternative route (Route $\mathrm{A}$ in Figure 1) is expected to capture some traffic currently using PR-153 and used as a substitute for the trips using PR-545.

\subsection{The Use of O-D Survey and SUE in Transportation Planning}

The use of O-D survey in transportation planning has been applied widely under various scenarios [4-9]. One of the previous studies which align with the objectives of this paper is the one conducted by Yang and Zhou [10] who highlighted that the quality of an estimated O-D matrix depends much on the reliability of the input data, and the number and locations of traffic counting points in the road network. They then addressed the problem of how to determine the optimal number and locations of traffic counting points in a road network for a given prior O-D distribution pattern. Origin and destination surveys can also be used for public transportation studies. $\mathrm{Hu}$ et al. [11] proposed the origin-destination of public transportation to help optimize layout of bus stops, reduce the influences of origin-destination of public transportation and improve the traffic efficiency of bus stations. They based their study on the nagel-schreckenberg traffic flow models and used the two-lane aggressive lane-changing rule to examine the influence of the origin-destination of the public transportation on the urban bidirectional four-lane mixed traffic flow. As for the O-D studies, user equilibriums has also been applied in different transportation planning studies [12-14]. For instance, Hazelton [15] indicated that the behavioral foundation of Stochastic User Equilibrium is that each traveler attempts to minimize

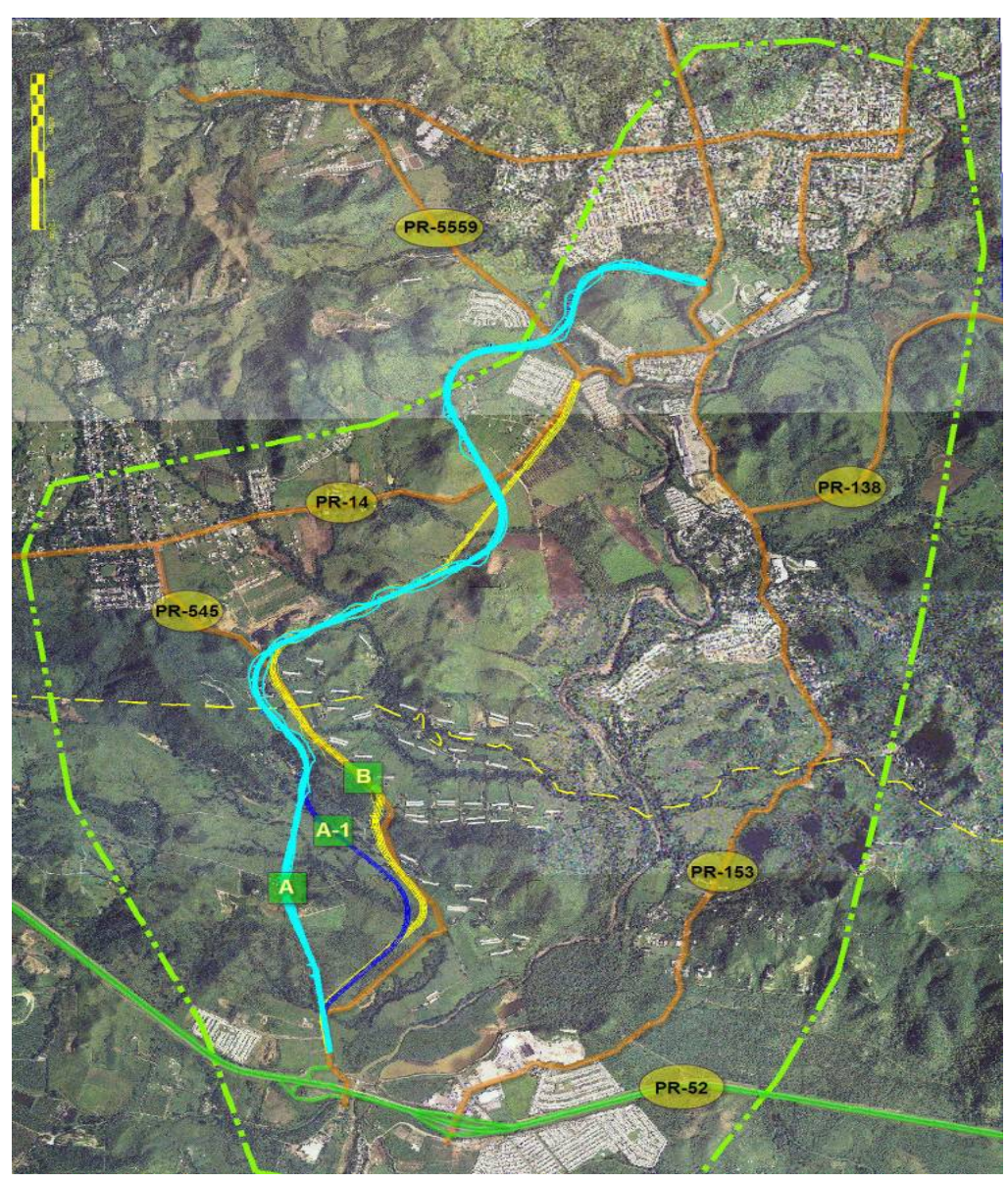

Figure 1. Town of Coamo existing road network. 
his or her perceived travel costs, where these costs are composed of a deterministic measured cost and a random term which can be interpreted as perceptual error. In his study, he presented Stochastic User Equilibrium as a probability distribution defined by the conditional route selection of each individual given the choices of all other travelers. He also investigated the limiting behavior as the travel demand becomes large. Some of the methodologies and procedures used in these previous studies which utilized O-D and SUE are replicated in this paper.

\subsection{Study Data and Methodology}

To achieve the project objectives, the study analyzed existing traffic condition for the PR-52, PR-153, PR-545 and PR-14. The 7-days, 24-hours count along these roadways were used to develop existing traffic characteristics. Historical traffic data, population growth, economic trends, employment growths and number of registered vehicles for the past years was used to develop the growth rates for traffic projection to the year 2027. The origin-destination survey was then conducted to determine major areas where the traffic enter and leave the Coamo town. The O-D provided the percentage of trips to and from the town of Coamo from different cities and zones, breakdown of trips by purpose, vehicle occupancy and classifications. Apart from revealing which highways were currently the major collectors and distributors to and from Coamo, the O-D study survey was also used to determine the possible future traffic pattern changes. The projected ADT (annual daily traffic) was portioned along PR-545 and PR-153 and the proposed new roadway based on the O-D survey results. As shown in Figure 1, Route A was proposed as an alternative for the traffic currently using PR-545 and those, which will be diverted from PR-153. Stochastic User Equilibrium trip assignment was used to distribute and balance the trips to and from Coamo along PR-153 and the proposed Route-A. Stochastic User Equilibrium (SUE) was used due to its underlying principle, which considers a population of drivers with homogeneous characteristics and perceive the same set of network costs except for the variation allowed by the stochastic choice model considered. With the fixed origin-destination within the known roadway networks, stochastic equilibrium assumes that the drivers will react to changes in network conditions as a result of change in route characteristics stochastically.

\section{Formulation of Origin-Destination Matrix and Findings}

As introduced earlier, Town of Coamo was the epicenter of this study; hence, all trips surveyed were coded with respect to routes to and from this town. Let $i$ denote town of Coamo, $j$ denote the external cities, and $n$ number of cities or separate routes to those cities, then

$$
\begin{gathered}
T_{i i}=\text { Internal Trips within Coamo } \\
T_{i j}=\text { Trips from Coamo to other cities } \\
T_{j i}=\text { Trips from other cities to Coamo }
\end{gathered}
$$

The proportion trip among all surveyed vehicles is given by;

Proportion of internal trips to total survey, $P_{i i}$;

$$
P_{i i}=\frac{T_{i i}}{\sum_{i=1}^{1} \sum_{j=1}^{n} T_{i j}+\sum_{j=1}^{n} \sum_{i=1}^{1} T_{j i}+\sum_{i=1}^{1} T_{i i}}
$$

Proportion of outbound external trips to total survey, $P_{i j}$;

$$
P_{i j}=\frac{T_{i j}}{\sum_{i=1}^{1} \sum_{j=1}^{n} T_{i j}+\sum_{j=1}^{n} \sum_{i=1}^{1} T_{j i}+\sum_{i=1}^{1} T_{i i}}
$$

Proportion of inbound external trips to total survey, $P_{j i}$;

$$
P_{j i}=\frac{T_{j i}}{\sum_{i=1}^{1} \sum_{j=1}^{n} T_{i j}+\sum_{j=1}^{n} \sum_{i=1}^{1} T_{j i}+\sum_{i=1}^{1} T_{i i}}
$$

Both trip proportions $P_{i i}, P_{i j}$ and $P_{j i}$ are utilized in determination of the trips along the existing and proposed routs.

The study was therefore initiated by preparing an O-D questionnaire for designated locations along PR-52, PR153 and PR-14. Different considerations were taken into account for effective O-D results including avoidance of uncertainties. One of the uncertainties avoided was to choose the interview locations which could have brought conflicting responses. According to some previous studies, the O-D location should consider traffic flow coverage and minimize the expected uncertainties [16,17]. Interviews were conducted on March 15, 2007 on PR 52 at the entrance and exit ramps with PR 153 and on PR 14 at the intersection with PR 153. The findings from O-D survey were summarized as

- PR 153 was the main highway from PR 52, used by motorist to and from Coamo,

- The intersection of PR 14/PR 153 was the major intersection used by motorist to/from Coamo,

- The PR 14 link from PR153 to downtown was the main receiver and deliverer of traffic from/to PR 153,

- Home, work and personal based trips were the major trip purposes to and from Coamo.

Figure 2 shows some of the results found from the O-D survey. For all of the trips to and from the town, $22 \%$ originate or ended west of Coamo, $13 \%$ south of Coamo and $13 \%$ east of Coamo.

\section{Proposed Alternative Route}

The 2027 projected ADT (Annual Daily Traffic) was 
33,800 vpd on PR-153 and 6200 vpd on PR-545. These ADT were taken as external trips. From the Origin Destination (O-D) matrix developed, it was found that of all external traffic to Coamo from PR-153, 46\% originated from the west, $28 \%$ from the south and $26 \%$ from the east. This distribution led to traffic assignment with respect to proposed route with 4-lane section and PR-153, which remained as a 2-lane section.

Table 1 elaborates the trip assignment developed based on origin destination survey analysis. Furthermore, from the survey it was observed that, out of $26 \%$ of the traffic going to Coamo from the east using PR 153, 11\% will be diverted to proposed Route A while 15\% will continue using PR-153 to Coamo. For the $28 \%$ of the traffic originating from the South to Coamo, 21\% will continue using PR-153 while 7\% will be diverted to Route A. Traffic from the west which make up $46 \%$ of external trips, 37\% was found that utilize Route A and 9\% PR-153. All traffic currently using PR-545 to downtown Coamo were assumed to be diverted to Route A. After all of the analysis using the O-D survey, PR 153 was found that will remain with 15,400 vehicles per day vpd which according to HCM is Level of Service (LOS) D, that was a reduction of $18,400 \mathrm{vpd}(54 \%)$ from originally 33,800 vpd projected. The proposed Route A was expected to receive $18,400 \mathrm{vpd}$ diverted from PR-153 and 6200 currently using PR-545, a total of 24,600 vpd (LOS B) by the year 2027. These O-D survey results were implemented in the Stochastic User Equilibrium analysis to find the final traffic balance based on the characteristics of existing PR-153 and the proposed Route A.

\section{Theory of User Equilibrium (UE) Stochastic User Equilibrium (SUE)}

User Equilibrium can be derived from Wardrop's first principle which states that, under equilibrium conditions traffic arranges itself in congested networks in such a way that no individual trip maker can reduce his travel cost by switching routes or all used routes between an origin and destination pair have equal and minimum costs while all unused routes have greater or equal costs $[14,18]$. A SUE condition is derived from the user equilibrium (UE) assumption which can be written as a given O-D pair as:

$$
f_{i}\left(c_{i}-u\right)=0 \text { for all } i
$$

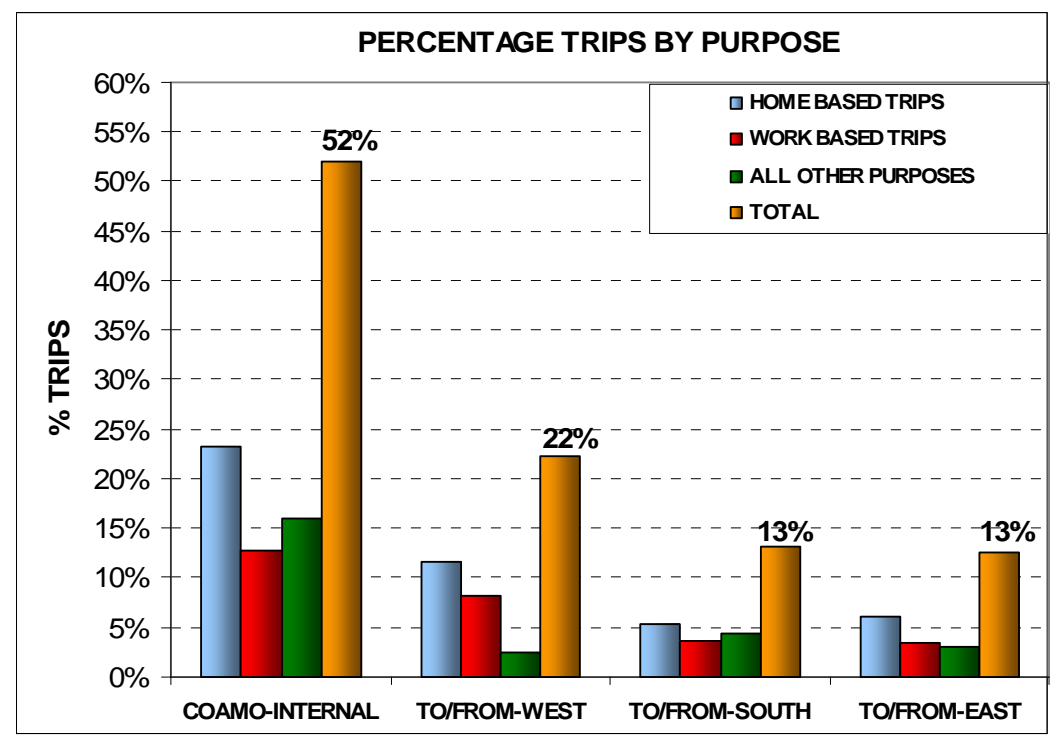

Figure 2. Percentage trips by location and purpose.

Table 1. Trip Assignment along PR-153 and new route using O-D survey results.

\begin{tabular}{ccccc}
\hline & \multicolumn{2}{c}{ To/From Coamo } & Through PR-153 & Through New-Route \\
\cline { 2 - 5 } & $\%$ & ADT & ADT & ADT \\
\cline { 2 - 5 } To/From East & $26 \%$ & 8900 & 5300 & 3600 \\
To/From South & $28 \%$ & 9300 & 7000 & 2300 \\
To/From West & $46 \%$ & 15,600 & 3100 & 12,500 \\
Pr-545 & & & 6200 \\
Total & $100 \%$ & 33,800 & 15,400 & 24,100 \\
\hline
\end{tabular}




$$
\begin{gathered}
\left(c_{i}-u\right)>0 \text { for all } i \\
\sum f_{i}=q \text { and } f_{i}>0
\end{gathered}
$$

where,

$f_{i}$ is the flow on Route $i$

$c_{i}$ is the travel cost on Route $i$

$u$ is the minimum cost

and $q$ is the combined flow.

Solution to the above conditions are obtained by solving an equivalent optimization program

$$
\operatorname{Min} \mathrm{z}=\sum_{i} \int_{0}^{v_{i}} t_{i}(v) \mathrm{d} v
$$

The assumptions regarding UE is include the road user having perfect knowledge of the route cost, travel time on a given link is a function of the flow on that link only and travel time functions being positive and increasing. The SUE extends the UE concept by considering the following assumptions and implications:

- Traveler has perfect knowledge of the network and travel cost,

- Traveler will choose the perceived least cost route,

- Different traveler perceives differently, thus introducing stochasticity.

The SUE route choice can then be analyzed using logit model, which treats all alternatives statistically independent. The formulation of SUE can be summarized as in Equation (4) [19]:

$$
\begin{aligned}
\operatorname{Min} z(v)= & -\sum q[\min c(v)] \\
& +\sum v_{i} t_{i}\left(v_{i}\right)-\sum_{i} \int_{0}^{v_{i}} t_{i}(\mathrm{v}) \mathrm{d} v
\end{aligned}
$$

where,

- The first term represent expected minimum route cost for all links,

- The second term represents expected total system travel time,

- The third term represents UE formulation,

- $z(v)=$ function for a given traffic volume $v$,

- $c(v)=$ route capacity for given traffic volume $v$,

- $v_{i}=$ traffic volume for route $i$,

- $t_{i}=$ travel time for route $i$,

- $\mathrm{d} v=$ change in traffic volume.

SUE utilizes multinomial models in stochastic assignment. Multinomial models use utilities which are independent and identically distributed mainly with a Gumbel distribution. They response homogeneity across individuals and there is error variance-covariance homogeneity across individuals.

\section{Application of SUE Coamo Route Relocation Study}

ADT volumes along PR-153 and Route A shown in Figure 3 . The travel times were formulated using posted speed limits, link lengths and signal density along each segment utilizing Bureau of Public Roads (BPR) [19].

$$
\begin{gathered}
t=t_{0}\left[1+a\left(\frac{V}{C}\right)^{b}\right] \\
t_{0}=\frac{L}{S_{0}}
\end{gathered}
$$

Combining Equations (5) and (6) gives;

$$
t=\frac{L}{S_{0}}\left[1+a\left(\frac{V}{C}\right)^{b}\right]
$$

where,

$t=$ link average travel time (hr),

$t_{0}=$ free flow link traversal time (hr),

$L=$ link length (mi),

$S_{0}=$ link Free-Flow Speed (FFS),

$V=$ link traffic volume in ADT,

$C=$ link ADT capacity,

$a$ and $b=$ The BPR function parameters are from Exhibit C30-1 and C30-2 of 2000 HCM [20].

The initial traffic flow for each link $V_{1}$ and $V_{2}$ were based on the O-D survey results discussed earlier. As shown in Figure 3, Route A is approximately 5 miles by length while PR-153 is approximately 5.5 miles. The first section of Route A from PR-52 is an uninterrupted flow while the second section which connects to Pr-14 to downtown is an arterial with two signalized intersection. Therefore signal density for Route A was taken as 0.5 per mile. PR-153 is an arterial with 4 signalized intersection making signal density to be 0.73 per mile. Posted speed limit for Route A is expected to be $55 \mathrm{mph}$ while for Pr-153 is $40 \mathrm{mph}$. Using exhibit C30-2 in $2000 \mathrm{HCM}$ [18], " $a$ " and " $b$ " values for each link were estimated as; $a=0.31$ and $b=3.64$ for Route A and $a=0.38$ and $b=$ 5.0 for PR-153

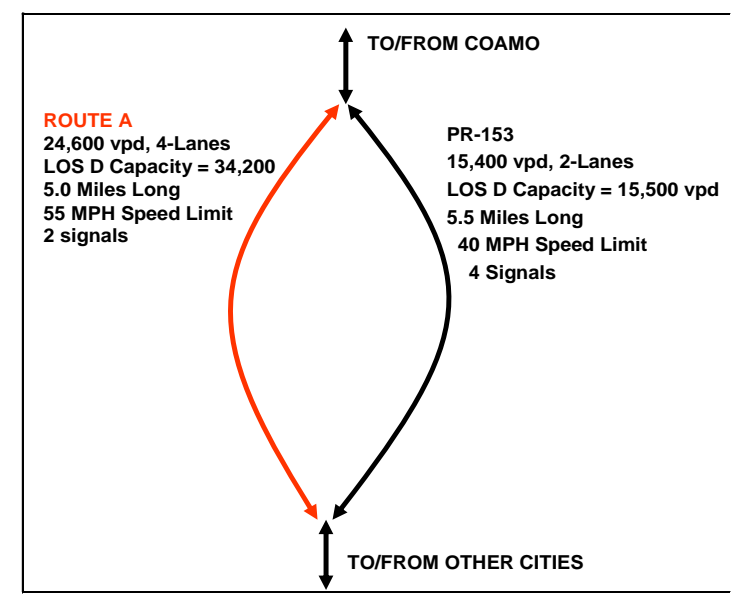

Figure 3. PR-153 and proposed Route A characteristics. 
By inserting these values in Equation (7) above yields the following formulations which are also illustrated in Figure 4:

Route A;

$$
t_{1}=0.091\left[1+0.31\left(\frac{V_{1}}{34200}\right)^{3.64}\right]
$$

with Initial $V_{1}=24,600 \mathrm{vpd}$

PR-153;

$$
t_{2}=0.138\left[1+0.38\left(\frac{V_{2}}{15500}\right)^{5.0}\right]
$$

with Initial $V_{2}=15,400 \mathrm{vpd}$.

\subsection{SUE Logit Utility Function}

The generalized cost functions used for each route are set based on theory developed by Kato et al. [20] using logit utility function in Equations (8) and (9).

The logit utility function which is formulated as;

$$
\begin{aligned}
& G C_{1}=\theta_{1} * t_{1}+\theta_{2} * \operatorname{Cost}+\theta_{3} *\left(\frac{v_{1}}{C_{1}}\right)^{2} * t_{1} \\
& G C_{2}=\theta_{1} * t_{2}+\theta_{2} * \operatorname{Cost}+\theta_{3} *\left(\frac{v_{2}}{C_{2}}\right)^{2} * t_{2}
\end{aligned}
$$

where

$G C_{1}=$ general Cost for Route 1 ,

$G C_{2}=$ general Cost for Route 2,

$t_{1}=$ travel time along Route $\mathrm{A}$,

$t_{2}=$ travel time along PR-153,

$\theta_{1}=$ In vehicle time constant coefficient, -0.094 ,

$\theta_{2}=$ Total Cost constant coefficient, -0.002 ,

$\theta_{3}=$ Congestion Index constant Coefficient, -0.009 ,

Cost $=$ in this study refers to gasoline consumption cost,

$v_{i}=$ traffic volume for route $i$,

$C_{i}=$ capacity for route $i$,

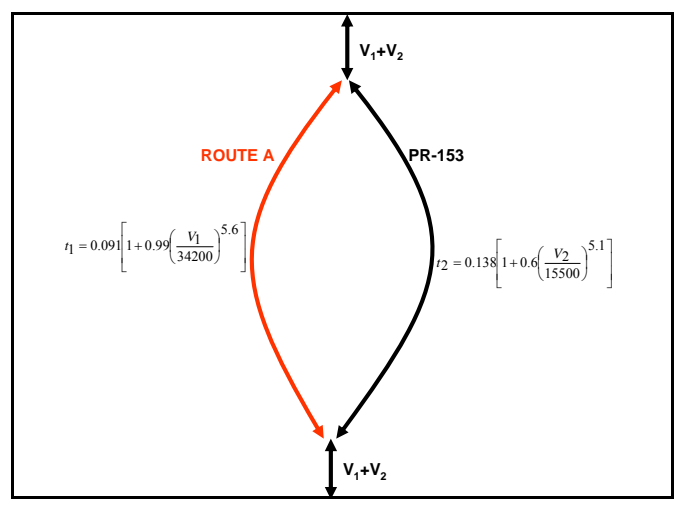

Figure 4. Link travel time and flow formulation along Route $A$ and PR-153.

$$
\left(\frac{v_{i}}{C_{i}}\right)^{2} * t_{i}=\text { Congestion Index }
$$

The value of coefficients $\theta_{1}, \theta_{2}$ and $\theta_{3}$ were adopted from previous researches by Kato et al. [6].

\subsection{Link Cost Due to Gasoline Expenses}

The "cost" which appears in the utility functions (8) and (9) refers to the situation where some travelers may choose the route based on the gasoline consumption. In this study, it is a cost with respect to gasoline consumption. The study compared Route A and PR-153 using the link length with respect to average fuel efficiency and price per gallon. The average fuel efficiency is taken 20 miles/gallon, while gasoline price was taken as $\$ 4.15$ / gallon (the gas price when the study was conducted). Therefore;

For Route A Link: length $=5.00$ miles,

Gasoline cost $=4.15 \times 5.00 / 20=\$ 1.04$ per link complete trip,

For PR-153 Link: length $=5.50$ miles,

Gasoline cost $=4.15 \times 5.50 / 20=\$ 1.14$ per link complete trip,

Probability of the traveler choosing Route A over PR-153 is

$$
P_{A}=\frac{\mathrm{e}^{-G C_{1}}}{\mathrm{e}^{-G C_{1}}+\mathrm{e}^{-G C_{2}}}
$$

Probability of the traveler choosing PR-153 over Route A is

$$
P_{153}=\frac{\mathrm{e}^{-G C_{2}}}{\mathrm{e}^{-G C_{1}}+\mathrm{e}^{-G C_{2}}}
$$

To determine the optimal balance volumes which take into account the described road characteristics and costs, the following conditions must be fulfilled;

$$
\begin{aligned}
& \left(V_{1}+V_{2}\right) * \mathrm{P}_{\mathrm{A}}-V_{1} \approx 0.0 \\
& \left(V_{1}+V_{2}\right) * \mathrm{P}_{153}-V_{2} \approx 0.0
\end{aligned}
$$

The outputs from Equation (12) gives the optimal traffic volume assignment and travel time by taking into account all variable and utility costs. The equation was programmed in Matlab for optimization and yielded;

\begin{tabular}{cccc}
\hline$G C_{1}$ & $G C_{2}$ & $V_{1}(\mathrm{vph})$ & $V_{2}(\mathrm{vph})$ \\
\hline-0.95 & -0.405 & 28,000 & 12,000 \\
\hline
\end{tabular}

Therefore, as a result of Stochastic User Equilibrium (SUE), the originally traffic assigned to Route $\mathrm{A}$ and PR-153 using O-D study percentage were optimized to the final assignments as follows: 


\begin{tabular}{cccc}
\hline & \multicolumn{3}{c}{ Traffic Assignment } \\
\cline { 2 - 4 } & From O-D Survey & From SUE & Deviation (\%) \\
\cline { 2 - 4 } Route A & 24,600 & 28,000 & $13.8 \%$ \\
PR-153 & 15,400 & 12,000 & $22 \%$ \\
\hline
\end{tabular}

\section{Conclusions}

The paper integrated the findings from origin-destination (O-D) survey and stochastic user equilibrium approach in route relocation study. The new route is proposed in the town of Coamo, Puerto Rico. The proposed new route is expected to capture diverted traffic currently using existing routes. The O-D provided the existing traffic pattern and characteristics with respect to trip purposes, and the percentages for internal and external trips. Percentages of trips from major cities surrounding the town were determined from the O-D survey. The initial trip assignments on the new and existing routes were based on the findings from O-D survey.

Stochastic User Equilibrium (SUE) was then applied to the network using the initial traffic volumes assigned from O-D survey findings. Included in the SUE was travel time on the links which is controlled by the free flow speed, maximum capacity, length of the link and signal spacing density. Apart from link travel time, the utility function of SUE contained other link measures of effectiveness such as time spent in the vehicle (in-vehicle time coefficient), congestion index and cost due to gasoline consumption (cost coefficient). The gasoline cost considered vehicle fuel efficient of 20 miles/gallon, gasoline price of $\$ 4.15 /$ gallon and length of the link. All these link characteristics were used to optimize the traveler choice of the route.

Traffic assignment from the SUE was slightly different from those initially assigned using O-D, indicating there was optimization. The assignment on new route was increased by $13.8 \%$ from the one assigned using O-D while assignment on the existing link was reduced by $22 \%$. The final optimized volumes were within capacity limits for each link indicating successful optimization. The final traffic assignment from SUE was used in the new route design. The findings from this study showed the possible benefit of integrating O-D with other trip assignment optimization approaches. By integrating O-D survey with optimization algorithms like UE or SUE can result in a well balanced links which take into account all possible constrains.

\section{REFERENCES}

[1] S. Jiang, B. Bai, G. Hong and J. O'Leary, "Understanding Travel Expenditure Patterns: A Study of Japanese Pleasure Travelers to the United States by Income Level," Jour- nal of Tourism Management, Vol. 25, No. 3, 2004, pp. 331-341. doi:10.1016/S0261-5177(03)00141-9

[2] H. Alibabai and H. S. Mahmassani, "Dynamic OriginDestination Demand Estimation Using Turning Movement Counts," The 87th Annual Meeting of the Transportation Research Board Proceedings, Washington DC, 2008.

[3] H. Spiess, "A Maximum Likelihood Model for Estimating Origin-Destination Matrices," Transportation Research, Vol. 21, No. 5, 1987, pp. 395-412. doi:10.1016/0191-2615(87)90037-3

[4] M. Hazelton, "Some Comments on Origin-Destination Matrix Estimation," Transportation Research Part A: Policy and Practice, Vol. 37, No. 10, 2003, pp. 811-822. doi:10.1016/S0965-8564(03)00044-2

[5] S. Han, "Dynamic Traffic Modeling and Dynamic Stochastic User Equilibrium Assignment for General Road Networks," Transportation Research Part B: Methodological, Vol. 37, No. 3, 2003, pp. 225-249. doi:10.1016/S0191-2615(02)00009-7

[6] M. Hazelton, "Estimation of Origin-Destination Matrices from Link Flows on Uncongested Networks," Transportation Research, Vol. 34, No. 7, 2000, pp. 549-566. doi:10.1016/S0191-2615(99)00037-5

[7] K. Jeornsten and S. Wallace, "Overcoming the (Apparent) Problem of Inconsistency in Origin-Destination Matrix Estimations," Transportation Science, Vol. 27, No. 4, 1993, pp. 374-380. doi:10.1287/trsc. 27.4 .374

[8] H. Lo., N. Zhang and W. Lam, "Estimation of an OriginDestination Matrix with Random Link Choice Proportions: A Statistical Approach," Transportation Research, Vol. 30, No. 4, 1996, pp. 309-324. doi:10.1016/0191-2615(95)00036-4

[9] M. Hazelton and M. Gordon, "Estimation of Origin-Destination Trip Matrices from Link Counts," Proceedings of the 2002 European Transport Conference, London, 2002.

[10] H. Yang and J. Zhou, "Optimal Traffic Counting Locations for Origin-Destination Matrix Estimation," Transportation Research Part B: Methodological, Vol. 32, No. 2, 1998, pp. 109-126. doi:10.1016/S0191-2615(97)00016-7

[11] J. Hu, L. Yang, L. Kong and Y. Yang, "Urban Mixed Traffic Flow Considering the Influence by Origin-Destination of Public Transportation," Journal of Transportation Systems Engineering and Information Technology, Vol. 11, No. 1, 2011, pp. 102-107. doi:10.1016/S1570-6672(10)60107-9

[12] S. Clark and P. Watling, "Sensitivity Analysis of the Probit-Based Stochastic User Equilibrium Assignment Model," Transportation Research Part B: Methodological, Vol. 36, No. 7, 2002, pp 617-635. doi:10.1016/S0191-2615(01)00021-2

[13] Y. Lim and B. Heydecker, "Dynamic Departure Time and Stochastic User Equilibrium Assignment," Transportation Research Part B: Methodological, Vol. 39, No. 2, 2005, pp. 97-118. doi:10.1016/j.trb.2003.08.003

[14] A. Nielsen, D. Frederiksen and N. Simonsen, "Stochastic User Equilibrium Traffic Assignment with Turn-Delays 
in Intersections," International Transactions in Operational Research, Vol. 5, No. 6, 1998, pp. 555-568. doi:10.1111/j.1475-3995.1998.tb00137.x

[15] M. Hazelton, "Some Remarks on Stochastic User Equilibrium," Transportation Research Part B: Methodological, Vol. 32, No. 2, 1998, pp. 101-108. doi:10.1016/S0191-2615(97)00015-5

[16] X. Fei and H. S. Mahmassani, "A Two-Stage Stochastic Model for the Sensor Location Problem in a Large-Scale Network," The 87th Annual Meeting of the Transportation Research Board Proceedings, Washington DC, 2008.

[17] K. N. Paul and H. K. L. William, "Evaluation of Count Location Selection Methods for Estimation of O-D Matrices," Journal of Transportation Engineering, Vol. 124, No. 4, 1998, pp. 376-383.

doi:10.1061/(ASCE)0733-947X(1998)124:4(376)
[18] Highway Capacity Manual, "Transportation Research Board," National Research Council, Washington DC, 2000.

[19] M. G. H. Bell and H. W. K. Lam, G. Ploss and D. Inaudi, "Stochastic User Equilibrium Assignment and Iterative Balancing," Proceedings of the 12th International Symposium on Transportation and Traffic Theory, Berkeley, 1993.

[20] S. Kato, Y. Kaneko and M. Inoue, "Empirical Analysis on Relationship Between Types of Travel Demand Techniques and Estimated User's Benefit Stemming from Transportation Investment," Journal of the Eastern Asia Society for Transportation Studies, Vol. 6, 2005, pp. 3937-3947. 